\title{
Aspectos Contemporâneos da Intervenção Coronária Percutânea no Tratamento Eletivo de Estenoses Localizadas no Tronco Não-Protegido da Artéria Coronária Esquerda
}

\author{
Guilherme Ferragut Attizzani', Hiram G. Bezerra'
}

\section{RESUMO}

A intervenção coronária percutânea (ICP) em tronco da artéria coronária esquerda (TCE) não-protegido era considerada até bem pouco tempo uma terapia proscrita em indivíduos elegíveis à revascularização cirúrgica convencional (RM), padrão de referência no tratamento dessa enfermidade. Esse conceito foi embasado nas elevadas taxas de eventos cardíacos adversos maiores em pacientes tratados com ICP com balão e, posteriormente, em pacientes tratados com stents não-farmacológicos. Mais recentemente, a introdução dos stents farmacológicos em nossa prática clínica diária promoveu redução substancial das taxas de reestenose e eventos cardíacos adversos maiores em pacientes tratados percutaneamente. Os bons resultados obtidos motivaram revisões das principais diretrizes, que passaram a considerar esse tratamento uma opção viável, variando seu nível de indicação conforme a complexidade clínica, bem como a anatomia coronária a ser abordada. Contudo, apesar desse importante avanço, alguns pontos devem ser destacados: a abordagem da bifurcação distal está associada a pior evolução em comparação ao tratamento do óstio ou corpo, sendo a técnica do T provisional a mais indicada nessa situação. Não foram estabelecidas, até o momento, diferenças substanciais nos desfechos entre os distintos stents farmacológicos, e seu implante, auxiliado por métodos de imagem intravasculares, deve ser incentivado. Finalmente, a avaliação e a seleção dos pacientes para o procedimento devem ser orientadas por meio de escores que avaliem características clínicas e angiográficas.

DESCRITORES: Angioplastia transluminal percutânea coronária. Stents farmacológicos. Vasos coronários.

\begin{abstract}
Contemporary Review of

Elective Percutaneous Coronary Intervention for Non-Protected Left Main Coronary Artery Disease

Percutaneous coronary intervention $(\mathrm{PCl})$ in the non-protected left main coronary artery (LM) was until recently considered a harmful practice for individuals eligible for coronary artery bypass grafting $(\mathrm{CABG})$, a gold standard in the treatment of this disease. This concept was based on the high rates of major adverse cardiac events in patients treated with balloon angioplasty and later on, in patients treated with bare-metal stents. More recently, the introduction of drugeluting stents in our daily clinical practice promoted marked reductions in the rates of restenosis and major adverse cardiac events in patients treated by $\mathrm{PCI}$. The good results obtained led to a review of the $\mathrm{PCl}$ guidelines, which now consider this treatment as a feasible option, classifying the level of indication according to clinical complexity as well as the coronary anatomy to be approached. However, despite this significant advance, it is important to highlight some aspects: treatment of the distal bifurcation is associated to a worse prognosis than ostial or midshaft lesions and the best approach in this situation seems to be the T-provisional technique. So far, substantial differences have not been established for different drug-eluting stents and intravascular imaging techniques should be strongly encouraged to guide their implant. Finally, patient assessment and selection for the procedure should be guided by scores based on clinical and angiographic characteristics.
\end{abstract}

KEY-WORDS: Angioplasty, transluminal, percutaneous coronary. Drug-eluting stents. Coronary vessels.

\footnotetext{
1 Harrington-McLaughlin Heart and Vascular Institute - Case Medical Center - Cleveland, Estados Unidos.

Correspondência: Guilherme Ferragut Attizzani. 11100 Euclid Avenue - Lakeside 3113 - Mailstop LKS 5038 - Cleveland, Ohio, USA 44106-5038

E-mail: gfattizzani@hotmail.com

Recebido em: 25/9/2010 • Aceito em: 21/11/2010
} 

no Tronco Não-Protegido da Artéria Coronária Esquerda. Rev Bras Cardiol Invasiva. 2010;18(3):456-67.

A artéria coronária esquerda é responsável, em média, por $75 \%$ da irrigação sanguínea do ventrículo esquerdo ${ }^{1}$ e a presença de obstruções significativas no tronco está associada a altos índices de morbidade e mortalidade. A cirurgia de revascularização do miocárdio vem sendo recomendada como padrão de referência no tratamento dessa enfermidade há três décadas, pois demonstrou reduzir a mortalidade a longo prazo quando comparada ao tratamento clínico. $^{2-5}$

A superioridade da cirurgia de revascularização do miocárdio em comparação à intervenção coronária percutânea no tratamento do tronco da artéria coronária esquerda foi demonstrada inicialmente na era préstent, ${ }^{6-8}$ e se manteve mesmo após o advento dos stents não-farmacológicos. Mais recentemente, a introdução dos stents farmacológicos na prática clínica foi responsável por redução significativa de eventos cardíacos adversos maiores, guiada, sobretudo, pela diminuição das taxas de novas revascularizações, em comparação aos stents não-farmacológicos. ${ }^{9-11}$ Adicionalmente, a ampliação do arsenal de fármacos disponíveis e a modernização das técnicas e dos demais materiais utilizados na intervenção coronária percutânea tornaram possível a abordagem bem-sucedida de lesões progressivamente mais complexas e com resultados que se mantêm a longo prazo. O objetivo da presente revisão é avaliar os aspectos contemporâneos da intervenção coronária percutânea em tronco da artéria coronária esquerda não-protegido, ou seja, aquele no qual não há um enxerto patente para a artéria descendente anterior ou artéria circunflexa.

\section{INTERVENÇÃO CORONÁRIA PERCUTÂNEA NO TRONCO DE CORONÁRIA ESQUERDA COM STENTS NÃO-FARMACOLÓGICOS}

As investigações iniciais acerca do uso de stents não-farmacológicos em tronco da artéria coronária esquerda foram marcadas por elevado sucesso técnico do procedimento, associado a baixas taxas de complicações intra-hospitalares. Entretanto, observou-se heterogeneidade nos resultados no seguimento tardio, consequência da variabilidade dos critérios de seleção dos pacientes e das técnicas empregadas. Adicionalmente, a ausência de estudos randomizados, com amostras consistentes para comparar a cirurgia de revascularização do miocárdio e a intervenção coronária percutânea, dificultou a avaliação da segurança e da eficácia do tratamento percutâneo nesse cenário. Os estudos disponíveis demonstram que a necessidade de revascularização do vaso-alvo variou de $4,8 \%$ a $23,9 \%$ e a mortalidade, de $2 \%$ a $24,2 \%$, de acordo com a complexidade clínica e angiográfica dos pacientes tratados percutaneamente (Tabela 1).

A maior complexidade dos pacientes do registro ULTIMA $^{17}(46 \%$ considerados não-elegíveis à cirurgia de revascularização do miocárdio) fez com que as taxas de mortalidade ao final de um ano se elevassem sobremaneira em relação às séries publicadas $(20,2 \%$ de mortalidade cardíaca). Os preditores independentes de mortalidade foram fração de ejeção $\leq 30 \%$, regurgitação mitral de graus 3 ou 4, apresentação clínica com infarto agudo do miocárdio associado a choque cardiogênico, creatinina $\geq 2 \mathrm{mg} / \mathrm{dl}$, além de calcificação grave da lesão. Contudo, o subgrupo de baixo risco (idade < 65 anos, fração de ejeção > 30\% e ausência de choque cardiogênico) demonstrou mortalidade em um ano de 3,4\%. A fração de ejeção reduzida foi identificada como sendo o preditor independente mais importante de óbito neste estudo, dado confirmado por Takagi et al. ${ }^{18}$.

A comparação de resultados de pacientes com lesão de tronco da artéria coronária esquerda tratados com stents não-farmacológicos aos resultados históricos do tratamento cirúrgico demonstra que tanto a mortalidade intra-hospitalar (0 e 2,3\%, respectivamente)

TABELA 1

Resultados da intervenção coronária percutânea em tronco da artéria coronária esquerda com uso de stents não-farmacológicos

\begin{tabular}{|c|c|c|c|c|}
\hline & Recorrência dos sintomas (\%) & RVA ou RLA (\%) & Reestenose $(\%)$ & Mortalidade (\%) \\
\hline Lopez et al. ${ }^{12}$ & ND & 13 & ND- & 2 \\
\hline Silvestri et al. ${ }^{13}$ & ND & 17,4 & ND & 8 \\
\hline Black Jr. et al. ${ }^{14}$ & ND & 4,8 & ND & $10,8 \neq$ \\
\hline Wong et al. ${ }^{15}$ & 20 & ND & ND & 2 \\
\hline Park et al. ${ }^{16}$ & ND & ND & 19 & 2,5 \\
\hline Tan et al. ${ }^{17}$ & ND & ND & ND & $24,2 \neq$ \\
\hline Takagi et al. ${ }^{18}$ & ND & 23,9 & 31,4 & 16,4 \\
\hline
\end{tabular}

₹ Mortalidade total.

ND = não disponível; RLA = revascularização da lesão-alvo; RVA = revascularização do vaso-alvo. 
como a mortalidade tardia $(3,2 \%$ em 5 anos e $4,5 \%$ em 3 anos, respectivamente) foram equivalentes. ${ }^{15,19} \mathrm{~A}$ Tabela 2 demonstra os resultados tardios da cirurgia de revascularização do miocárdio no tratamento da lesão de tronco da artéria coronária esquerda.

Com base nesses resultados, a indicação da intervenção coronária percutânea com stents não-farmacológicos em tronco da artéria coronária esquerda ficou restrita apenas àqueles indivíduos de elevado risco cirúrgico, sendo formalmente contraindicada nos demais cenários clínicos. ${ }^{26}$

\section{INTERVENÇÃO CORONÁRIA PERCUTÂNEA NO TRONCO DE CORONÁRIA ESQUERDA NÃO-PROTEGIDO COM STENTS FARMACOLÓGICOS}

Os stents farmacológicos são seguros e eficazes no tratamento da doença arterial coronária. Embora não tenhamos dados robustos demonstrando o impacto na sobrevida, esses dispositivos se impuseram na prática clínica pela redução substancial das taxas de reestenose e de novas revascularizações, quando comparados aos stents não-farmacológicos. ${ }^{27-31}$ A possibilidade de reduzir a reestenose, que nem sempre se manifesta de modo benigno como se pensava inicialmente ${ }^{32,33}$ e que nesta particular localização anatômica pode se associar a morbidade ainda mais elevada, entusiasmou investigadores a avaliar o uso de stents farmacológicos nas lesões de tronco da artéria coronária esquerda.

\section{Primeiros estudos}

As investigações iniciais acerca do uso dos stents farmacológicos na intervenção coronária percutânea em tronco da artéria coronária esquerda não-protegido foram realizadas com número reduzido de pacientes, observando-se as mesmas taxas de sucesso do procedimento obtidas com os stents não-farmacológicos, acrescentando-se redução significativa de desfechos ad- versos no seguimento tardio. Em registro conduzido por de Lezo et al. ${ }^{34}$, foram demonstradas taxas de revascularização do vaso-alvo de apenas $2 \%$. Chieffo et al. ${ }^{35}$ trataram 85 pacientes de alto risco $(81,2 \%$ com envolvimento da bifurcação distal, 45\% com EuroSCORE > 6), comparando-os a dados históricos de 64 indivíduos tratados com stents não-farmacológicos, destacando redução de $73 \%$ da ocorrência de eventos cardíacos adversos maiores. Adicionalmente, esse estudo demonstrou que todos os casos de reestenose do grupo que recebeu stents farmacológicos ocorreram em indivíduos nos quais foi abordada a bifurcação do tronco da artéria coronária esquerda.

Park et al. ${ }^{36}$ realizaram intervenção coronária percutânea em tronco da artéria coronária esquerda nãoprotegido em 102 pacientes portadores de função ventricular preservada, auxiliados pelo uso do ultrassom intracoronário e abordando a bifurcação distal em 70\% dos casos. As taxas de reestenose obtidas aos 6 meses foram significativamente menores quando comparadas aos dados históricos de 121 indivíduos tratados com stents não-farmacológicos (7\% e 30,3\%; P < 0,001) e a revascularização da lesão-alvo ao final de um ano de seguimento ocorreu em $2 \%$ e $17,4 \%$, respectivamente $(\mathrm{P}<0,001)$. O registro multicêntrico observacional e retrospectivo GISE-SICl ${ }^{37}$ fez comparação semelhante em 1.453 pacientes, com a particularidade de excluir aqueles nos quais foi abordada a bifurcação distal, obtendo números equivalentes, nos 479 pacientes analisados, para revascularização da lesão-alvo $(P=0,6)$ bem como para infarto agudo do miocárdio ou eventos cardíacos adversos maiores ao final de um acompanhamento de três anos. Observou-se inesperada vantagem do uso de stents farmacológicos em termos de redução de mortalidade [hazard ratio (HR) 0,37, intervalo de confiança de 95\% (IC 95\%) 0,15-0,96; P = 0,04); contudo, devemos interpretar esses resultados com cautela, por não se tratar de um estudo prospectivo e randomizado.

TABELA 2

Resultados de estudos que avaliaram a cirurgia de revascularização do miocárdio no tronco da artéria coronária esquerda (adaptado de Taggart et al. ${ }^{20}$ )

\begin{tabular}{|c|c|c|c|c|c|c|}
\hline Autor & Período & $\mathbf{n}$ & $\begin{array}{l}\text { Mortalidade } \\
\text { hospitalar } \\
(\%)\end{array}$ & $\begin{array}{c}\text { Mortalidade } \\
\text { aos } 30 \text { dias } \\
(\%)\end{array}$ & $\begin{array}{c}\text { Mortalidade } \\
\text { em } 1 \text { ano } \\
(\%)\end{array}$ & $\begin{array}{c}\text { Mortalidade } \\
\text { aos } 2 \text { anos } \\
(\%)\end{array}$ \\
\hline Ellis et al. ${ }^{19}$ & 1990-1995 & 1.585 & 2,3 & ND & ND & ND \\
\hline Jonsson et al. ${ }^{21}$ & 1970-1999 & 1.888 & 2,7 & ND & ND & ND \\
\hline Lu et al. ${ }^{22}$ & 1997-2003 & 1.197 & 2,8 & 3 & 5 & 6 \\
\hline Keogh e Kinsman ${ }^{23}$ & 2003 & 5.003 & 3 & ND & ND & ND \\
\hline Dewey et al. ${ }^{24}$ & 1998-1999 & 728 & ND & 4,2 & ND & ND \\
\hline Yeatman et al. ${ }^{25}$ & 1996-2000 & 387 & 2,4 & ND & ND & 5 \\
\hline
\end{tabular}

ND = não disponível. 

no Tronco Não-Protegido da Artéria Coronária Esquerda. Rev Bras Cardiol Invasiva. 2010;18(3):456-67.

\section{Bifurcação distal: o "calcanhar de Aquiles" da intervenção coronária percutânea em tronco da artéria coronária esquerda}

O tratamento percutâneo de lesões localizadas nas bifurcações das artérias coronárias é, em geral, complexo e historicamente tem sido relacionado a maiores taxas de insucesso bem como de eventos adversos ao longo do seguimento, quando comparado à intervenção coronária percutânea em lesões não-bifurcadas. ${ }^{38}$ O uso rotineiro de dois stents nesse cenário aparentemente não é vantajoso quando comparado ao uso de apenas um stent no ramo principal e implante no ramo lateral apenas em caso de resultado não satisfatório (provisional stenting), e associa-se a aumento do tempo do procedimento, maior quantidade de contraste, aumento dos marcadores de necrose miocárdica e taxas de má-expansão e má-aposição significativas, sobretudo quando considerado o óstio do ramo lateral. ${ }^{39-42}$

Especificamente em relação às lesões que acometem a bifurcação do tronco da artéria coronária esquerda, Biondi-Zoccai et al. ${ }^{43}$ publicaram meta-análise que envolveu 1.278 indivíduos seguidos, em média, por período de 10 meses, na qual creditaram aos stents farmacológicos redução de $66 \%$ do risco relativo de eventos cardíacos adversos maiores quando comparados aos não-farmacológicos, além de promoverem decréscimo da ordem de $54 \%$ no desfecho composto de eventos cardíacos adversos maiores e acidente vascular encefálico comparativamente à cirurgia de revascularização do miocárdio. O envolvimento da bifurcação distal foi identificado como preditor independente de eventos cardíacos adversos maiores $(P=0,001)$ e de revascularização do vaso-alvo $(P=0,020)$, resultados concordantes com publicações prévias de Valgimigli et al. ${ }^{44}$ e Godino et al. ${ }^{45}$, que salientaram maior eficácia do tratamento de lesões localizadas no óstio e na porção média do tronco da artéria coronária esquerda.

O registro J-CYPHER ${ }^{46}$ corroborou esses dados, ressaltando em seu seguimento de 3 anos uma evolução desfavorável em termos de revascularização da lesão-alvo dos indivíduos com acometimento da bifurcação distal do tronco da artéria coronária esquerda tratados com stents eluidores de sirolimus, em comparação àqueles cujas lesões se localizavam no óstio ou corpo $(17,1 \%$ vs. $3,6 \% ; P=0,005)$; contudo, as taxas de mortalidade cardíaca mostraram-se equivalentes $(7,6 \%$ vs. $9,8 \% ; P=0,41)$. Os indivíduos que receberam o implante de dois stents em comparação com um stent nesse estudo exibiram maior mortalidade cardíaca e revascularização da lesão-alvo.

Price et al. ${ }^{47}$ também contribuíram para a elucidação da complexidade do tratamento da bifurcação distal do tronco da artéria coronária esquerda por meio da avaliação de 50 indivíduos cuja mortalidade, de apenas $2 \%$ no seguimento de 9 meses, foi ofuscada por taxas de revascularização da lesão-alvo de $38 \%$.
Em estudo de Palmerini et al. ${ }^{48}$, no qual foram incluídos 773 pacientes, o implante de uma única endoprótese foi associado a menores taxas de desfechos adversos no seguimento de 2 anos quando comparado ao uso rotineiro de duas endopróteses, confirmando, desse modo, os resultados obtidos previamente por Kim et al. ${ }^{49}$, em que o uso de dois stents esteve associado a taxas mais elevadas de reestenose $(24,4 \%$ e $5,3 \%$, respectivamente, para o uso de dois stents e de um stent; $P=0,024)$, levando a uma sobrevida livre de revascularização da lesão-alvo de $86 \%$ e $100 \%(P=0,004)$ ao final de 18 meses.

Mais recentemente, registro francês que utilizou stents eluidores de paclitaxel e avaliou 291 pacientes com envolvimento da bifurcação distal tratados pela técnica do T provisional, seguidos por 2 anos, ressaltou a segurança e a eficácia dessa abordagem, com mortalidade cardíaca de 5,4\%, revascularização da lesão-alvo de $8,7 \%$ e trombose definitiva ou provável de $0,7 \% .^{50}$

\section{Análise dos resultados do registro MAIN-COMPARE}

A abordagem percutânea em tronco da artéria coronária esquerda ganhou força com a publicação do registro multicêntrico $M A I N-C O M P A R E^{51}$, que incluiu 2.240 pacientes consecutivos e comparou o tratamento do tronco da artéria coronária esquerda por meio da cirurgia de revascularização do miocárdio ou da intervenção coronária percutânea (28,9\% receberam stents não-farmacológicos e 71,1\% receberam stents farmacológicos, dos quais $77,4 \%$ eram eluidores de sirolimus e $22,6 \%$, eluidores de paclitaxel), não mostrando diferenças significativas nos desfechos de segurança avaliados. Contudo, a cirurgia de revascularização do miocárdio revelou-se mais eficaz na redução de taxas de revascularização do vaso-alvo ao final do seguimento de 1.017 dias, quando comparada ao tratamento tanto com stents não-farmacológicos (HR 10,7, IC 95\% 3,8-29,9; P < 0,001) como com farmacológicos (HR 5,96, IC 95\% 2,5-14,1; P < 0,001).

Os dados coletados nesse registro coreano, de pacientes não-selecionados, permitiram gerar subestudo que comparou, ao longo de 3 anos, os pacientes que receberam implante de stents farmacológicos $(n=864)$ e não-farmacológicos ( $n=353)$, e não encontrou diferenças significativas em termos de mortalidade ou infarto agudo do miocárdio. O risco de revascularização da lesão-alvo foi reduzido com os stents farmacológicos, comparativamente aos não-farmacológicos (5,4\% e $12,1 \%$, respectivamente; HR 0,40, IC 95\% 0,22-0,73; $\mathrm{P}=0,003)$. Quando se avaliam os resultados de acordo com a localização anatômica da lesão, verificam-se melhores resultados nas lesões de óstio e corpo do tronco da artéria coronária esquerda (Tabela 3). ${ }^{52}$

A influência do diabetes nos resultados da cirurgia de revascularização do miocárdio e da intervenção 
TABELA 3

Resultados do registro MAIN-COMPARE ${ }^{22}$ comparando o uso de stents farmacológicos e não-farmacológicos em diferentes localizações anatômicas no tronco da artéria coronária esquerda

\begin{tabular}{lcccccc}
\hline & $\begin{array}{c}\text { SF } \\
\text { (óstio/corpo) }\end{array}$ & $\begin{array}{c}\text { SNF } \\
\text { (óstio/corpo) }\end{array}$ & $\mathbf{P}$ & $\begin{array}{c}\text { SF } \\
\text { (bifurcação) }\end{array}$ & $\begin{array}{c}\text { SNF } \\
\text { (bifurcação) }\end{array}$ & P \\
\hline Mortalidade, \% & 8,8 & 8,7 & 0,97 & 7,6 & 10,9 & 0,23 \\
Mortalidade ou IAM, \% & 12,4 & 13,1 & 0,64 & 15,7 & 22,6 & 0,08 \\
Revascularização da lesão-alvo, \% & 3,4 & 10,3 & $<0,001$ & 6,9 & 16,3 & 0,001 \\
\hline
\end{tabular}

IAM = infarto agudo do miocárdio; SF = stents farmacológicos; SNF = stents não-farmacológicos.

coronária percutânea com stents farmacológicos também foi investigada nesse registro, destacando-se a semelhança nos desfechos de segurança e menor eficácia da intervenção coronária percutânea em relação à revascularização do vaso-alvo (HR 4,31, IC 95\% 2,28-8,15; $\mathrm{P}<0,001) .{ }^{53}$

Recentemente, os resultados do acompanhamento de 5 anos do estudo MAIN-COMPARE demonstraram a equivalência de resultados entre os grupos de revascularização miocárdica e intervenção coronária percutânea na maior parte dos desfechos, à exceção da revascularização do vaso-alvo, que se manteve mais elevada no grupo de intervenção coronária percutânea (HR 5,11; IC 95\% 3,52-7,42; P $<0,001) . .^{54}$

\section{Mais contribuições originais e meta-análises}

Outro ensaio clínico que avaliou a intervenção coronária percutânea em tronco da artéria coronária esquerda foi conduzido por Han et al. ${ }^{55}$, que ressaltaram as vantagens previamente descritas dos stents farmacológicos em comparação aos não-farmacológicos, em termos de melhora dos desfechos de eficácia. Resultado semelhante foi observado no subgrupo de pacientes submetidos a intervenção coronária percutânea com stents farmacológicos e não-farmacológicos no estudo LE MANS ${ }^{56}$, que, em seguimento a longo prazo (3,8 anos), apresentou redução de eventos cardíacos adversos maiores $(14,9 \%$ e $25,9 \%$; $P=0,039)$ bem como de taxas de revascularização da lesão-alvo (3,2\% e $10,1 \% ; P=0,04)$. Sanmartín et al. ${ }^{57}$ também avaliaram o desempenho das endopróteses eluidoras de medicamentos no tronco da artéria coronária esquerda em um registro unicêntrico, e verificaram que, em comparação ao tratamento cirúrgico, houve comportamento semelhante em termos da ocorrência de eventos cardíacos adversos maiores, mas menores taxas de novas revascularizações no grupo submetido a revascularização miocárdica $(0,8 \%$ vs. 5,2\%; P = 0,02).

Recentemente, duas importantes meta-análises compararam o uso dos stents farmacológicos à cirurgia de revascularização do miocárdio e aos stents não-farmacológicos no tratamento da lesão de tronco da artéria coronária esquerda. Na primeira, que englobou 3.773 pacientes, houve vantagens da cirurgia apenas em termos de redução de novas revascularizações. ${ }^{58}$ Já na segunda, que incluiu 44 estudos e 10.342 pacientes, foram relatadas, ao final de 3 anos de seguimento, vantagens significativas da utilização de stents farmacológicos, em comparação aos stents não-farmacológicos, em termos de redução de mortalidade, infarto agudo do miocárdio, novas revascularizações e eventos cardíacos adversos maiores. ${ }^{59}$

\section{Estudos randomizados, seguimentos a longo prazo e segurança}

Erglis et al. ${ }^{60}$ compararam, em pequeno estudo randomizado, o uso de stents eluidores de paclitaxel ao uso de stents não-farmacológicos, confirmando a redução das taxas de reestenose obtidas nos registros previamente descritos ( $6 \%$ e $22 \%$, respectivamente; $P=0,021)$, além de exibirem melhor desempenho na sobrevida livre de eventos cardíacos adversos maiores ao longo do seguimento de 6 meses.

O ensaio clínico LE MANS ${ }^{61}$ foi pioneiro em comparar, de forma randomizada, a cirurgia de revascularização do miocárdio e a intervenção coronária percutânea em tronco da artéria coronária esquerda (35\% de uso de stents farmacológicos), obtendo resultados favoráveis ao tratamento percutâneo em relação a seu desfecho primário, a melhora da fração de ejeção do ventrículo esquerdo $(0,5 \pm 0,8 \%$ e $3,3 \pm 6,7 \%$; $P=0,047)$. Ao final de um ano de seguimento, as taxas de sobrevida mostraram-se equivalentes $(92,5 \%$ e $98,1 \%$; $P=0,37)$, assim como a sobrevida livre de eventos cardíacos adversos maiores $(75,5 \%$ e $71,2 \%$; $P=0,29)$, apesar da maior taxa de nova revascularização do tratamento percutâneo [risco relativo (RR) 1,27, IC 95\% 1,05-1,54; $P=0,01]$.

A mais importante contribuição recente foi fornecida pelo estudo SYNTAX ${ }^{62}$, no qual 705 pacientes com lesão de tronco da artéria coronária esquerda foram randomizados para intervenção coronária percutânea com stents eluidores de paclitaxel ou cirurgia de revascularização do miocárdio. Seus resultados demonstraram desempenho similar dos dois tratamentos em termos de mortalidade ou infarto agudo do miocárdio no 
Attizzani GF, et al. Aspectos Contemporâneos da Intervenção Coronária Percutânea no Tratamento Eletivo de Estenoses Localizadas no Tronco Não-Protegido da Artéria Coronária Esquerda. Rev Bras Cardiol Invasiva. 2010;18(3):456-67.

seguimento de um ano e menor necessidade de novas revascularizações $(11,8 \%$ e $6,5 \% ; P=0,02)$, mas maior ocorrência de acidentes vasculares encefálicos $(0,3 \%$ e $2,7 \% ; P=0,009)$ no grupo cirúrgico.

Na comparação de três subgrupos distintos de pacientes, de complexidade anatômica progressivamente mais elevada, as taxas de eventos cardíacos adversos maiores mostraram-se equivalentes nos indivíduos de menor risco, enquanto naqueles cuja anatomia se mostrou menos favorável houve pior desempenho da intervenção coronária percutânea em comparação ao grupo submetido a revascularização do miocárdio (25,3\% e $12,9 \% ; P=0,008)$, sobretudo em decorrência das taxas mais elevadas de revascularização do vaso-alvo. Esses dados foram fortalecidos pela apresentação dos resultados de 2 anos de seguimento desses pacientes, em que se confirmou a ausência de diferenças significativas entre os grupos em termos de desfechos de segurança como mortalidade ou infarto agudo do miocárdio; entretanto, as taxas de novas revascularizações mantiveram a tendência inicial (17,3\% vs. 10,4\% para intervenção coronária percutânea e cirurgia de revascularização do miocárdio, respectivamente; $P=0,01){ }^{63}$

No que diz respeito à segurança dessa intervenção, destacam-se as baixas taxas de trombose do stent. Godino et al. ${ }^{45}$ encontraram $1,1 \%$ de trombose definitiva no seguimento de 4 anos; Valgimigli et al. ${ }^{64}$ encontraram $0,9 \%$ de trombose e $2,4 \%$ de mortalidade cardíaca ou infarto agudo do miocárdio ao longo de um ano; Chieffo et al. ${ }^{65}, 0,9 \%$ de trombose definitiva ou provável no seguimento de 30 meses; e, finalmente, Mehilli et al. ${ }^{66}, 1 \%$ de trombose definitiva em um ano de seguimento. Adicionalmente, no que concerne ao manejo da reestenose após o implante de stents farmacológicos no tronco da artéria coronária esquerda, verificamos que a maioria foi tratada com nova intervenção coronária percutânea, mantendo-se baixas as taxas de trombose após a reintervenção. ${ }^{67}$

\section{Escolha da endoprótese}

Praticamente todos os estudos nos quais se utilizaram stents farmacológicos em nossa análise incluíram as endopróteses eluidoras de sirolimus ou paclitaxel. O registro SP-DELFT ${ }^{68}$ não estabeleceu diferenças entre elas quando avaliadas as taxas de eventos cardíacos adversos maiores, salientando baixas taxas de trombose $(0,3 \%)$ ao final de 3 anos de seguimento. Esses dispositivos também foram comparados no estudo ISARLEFT-MAIN ${ }^{66}$, que incluiu 607 pacientes (35\% EuroSCORE $\geq 6$ ), nos quais a bifurcação distal foi abordada em $63 \%$ das intervenções. O desfecho primário de mortalidade, infarto agudo do miocárdio ou revascularização da lesão-alvo ocorreu em $13,6 \%$ dos indivíduos do grupo que recebeu stents eluidores de paclitaxel e em $15,8 \%$ dos que receberam stents eluidores de sirolimus (RR 0,85, IC 95\% 0,56-1,29; P = 0,44) no seguimento de um ano. As taxas de trombose foram similares entre os grupos e não atingiram a marca de 1\%. Ao final de 2 anos de seguimento, observaram-se taxas de mortalidade de $10,7 \%$ e $8,7 \%$, respectivamente, para os grupos paclitaxel e sirolimus (RR 1,14, IC 95\% 0,66-1,95; $\mathrm{P}=0,64)$, e a reestenose angiográfica ocorreu em $16 \%$ e $19,4 \%$, respectivamente $(P=0,3)$. Praticamente todos os casos de reestenose, à exceção de uma localizada no óstio, ocorreram na bifurcação distal do tronco da artéria coronária esquerda, reforçando os dados de pior evolução desse sítio anatômico apresentados previamente.

Há carência de informações na literatura acerca do uso de outros tipos de stents farmacológicos. Em análise prospectiva conduzida por Nakamura et al. ${ }^{69}$, que incluiu 676 pacientes, o uso de stents eluidores de everolimus, sirolimus ou biolimus foi associado à redução de taxas de reestenose e de novas revascularizações no seguimento de 9 meses quando comparado ao uso de stents eluidores de paclitaxel ou zotarolimus. Adicionalmente, os resultados satisfatórios publicados recentemente demonstrando equivalência ${ }^{70}$ e até superioridade ${ }^{71}$ de algumas dessas novas endopróteses em relação às de primeira geração, em distintas apresentações clínicas, nos levam a especular que brevemente esses resultados serão reproduzidos em maior escala também no tronco da artéria coronária esquerda.

\section{Uso de métodos de imagem auxiliares intracoronários}

O uso do ultrassom intracoronário para guiar a intervenção coronária percutânea leva à expansão mais efetiva e, consequentemente, à obtenção de maiores áreas luminais mínimas dos stents implantados quando comparado ao implante guiado exclusivamente pela angiografia. ${ }^{72}$ Contudo, muitas das investigações realizadas com stents não-farmacológicos com a utilização dessa modalidade de imagem para a otimização do procedimento utilizaram técnicas subótimas ou não tinham tamanho da população suficiente para alcançar poder estatístico para demonstrar vantagens em termos de redução de desfechos clínicos adversos, ${ }^{73,74}$ tornando sua aplicação rotineira incerta.

$\mathrm{Na}$ era dos stents farmacológicos, recentes publicações sugerem benefícios do implante guiado por ultrassom intracoronário em termos de redução de novas revascularizações e trombose dos stents, ${ }^{75}$ bem como da otimização do resultado final do procedimento, demonstrado pela obtenção de maiores áreas luminais mínimas. ${ }^{76}$ Assim sendo, intuitivamente podemos considerar que a realização de um implante otimizado de stent no tronco da artéria coronária esquerda guiado pelo ultrassom intracoronário seria de particular importância, visto a grande área de miocárdio em risco, minimizando a ocorrência de má-expansão, má-aposição ou de cobertura incompleta da lesão, fatores descritos previamente como preditores de falência do vaso-alvo. ${ }^{77}$ 

no Tronco Não-Protegido da Artéria Coronária Esquerda. Rev Bras Cardiol Invasiva. 2010;18(3):456-67.

Recentemente, o maior estudo relacionado a esse tema incluiu 975 pacientes, tendo demonstrado tendência a menor mortalidade no seguimento de 3 anos no grupo em que a intervenção coronária percutânea em tronco da artéria coronária esquerda foi orientada pelo ultrassom intracoronário, quando comparada à guiada exclusivamente pela angiografia (6\% e 13,6\%, respectivamente; $\mathrm{P}=0,063)$, sobretudo nos indivíduos em que foram utilizados stents farmacológicos $(4,7 \%$ e $16 \%$, respectivamente; $\mathrm{P}=0,048)$. Esse benefício não foi observado nos indivíduos que receberam implante de stents não-farmacológicos, nem houve redução de taxas de infarto agudo do miocárdio ou de revascularização do vaso-alvo nesse grupo. ${ }^{78}$

Outra ferramenta intravascular emergente, a tomografia por convergência óptica, dispositivo de imagem dotado de alta resolução axial $(\sim 10 \mu \mathrm{m})$, permite avaliação luminal minuciosa, em especial da aposição, da expansão e da cobertura das hastes dos stents implantados, além de detectar a presença de prolapso de placa e de tecido intraluminal anormal. ${ }^{79}$ A limitação inicial do uso dessa ferramenta nas lesões de tronco da artéria coronária esquerda decorreu sobretudo da necessidade de oclusão proximal do vaso investigado para a realização do procedimento; contudo, novos aparelhos, denominados Fourier-Domain, permitem que a tomografia por convergência óptica seja realizada sem esse inconveniente ${ }^{80}$, tornando possível sua avaliação no tratamento percutâneo das lesões de tronco da artéria coronária esquerda.

\section{Estudos com acompanhamento muito tardio}

Apesar do avanço inquestionável experimentado pela intervenção coronária percutânea em tronco da artéria coronária esquerda, alguns resultados recentemente apresentados, relativos ao seguimento a longo prazo, demonstram que é necessário cautela nas indicações, respeitando o perfil clínico e angiográfico dos pacientes.

O primeiro estudo relata o seguimento de até 4 anos de 148 pacientes que receberam implante de stents eluidores de sirolimus e paclitaxel no Thoraxcenter, em Roterdã, Holanda, no qual Onuma et al. ${ }^{81}$ chamam a atenção para o aumento relativo de $58 \%$ dos eventos cardíacos adversos maiores e a elevação das taxas de mortalidade total de $19,6 \%$ para $33,1 \%$, comparandose o primeiro ao quarto anos de seguimento. Os autores, no entanto, ressaltaram que o perfil de risco dos indivíduos incluídos foi elevado, incluindo-se 24\% dos casos em vigência de infarto agudo do miocárdio com supra de ST e escore SYNTAX médio de 40.

Já a experiência de Milão, relatada por Chieffo et al. ${ }^{82}$, incluiu 249 pacientes com menor risco (escore SYNTAX médio de 29) e comparou a intervenção coronária percutânea com stents farmacológicos à cirurgia de revascularização do miocárdio. A análise dos resultados demonstrou mortalidade ao longo de 5 anos equivalente entre os grupos $(15,9 \%$ e $18,3 \%$, respecti- vamente) e melhor desempenho da cirurgia de revascularização do miocárdio em relação à necessidade de novas revascularizações. Apesar das mortalidades semelhantes, as taxas de trombose dos stents encontradas, de cerca de $6 \%(0,93 \%$ definitiva ou provável e 5,6\% possível), preocupam.

Na Tabela 4 estão apresentados estudos com a utilização de stents farmacológicos na intervenção coronária percutânea em tronco da artéria coronária esquerda.

\section{SELEÇÃO DOS PACIENTES}

A seleção dos pacientes a serem tratados é um aspecto de particular importância nessa situação. $O$ uso do escore SYNTAX, que avalia as características anatômicas da árvore coronária, auxilia na predição de eventos cardíacos adversos maiores e de mortalidade nesses pacientes. ${ }^{86}$ Pacientes com escore $>34$ mostraram benefício com o tratamento cirúrgico, em termos de redução da mortalidade. ${ }^{87}$

Adicionalmente, na tentativa de refinar a seleção de pacientes, podemos agregar o EuroSCORE, que avalia características clínicas, estratégia adotada por Capodanno et al. ${ }^{88}$, que demonstraram melhora da predição de mortalidade cardíaca com a associação dos escores, em comparação à aplicação isolada de ambos. Recentemente, Chen et al. ${ }^{89}$ compararam a utilização do escore SYNTAX ao escore NERS, que engloba características angiográficas, clínicas e do procedimento, na predição de eventos adversos na intervenção coronária percutânea em tronco da artéria coronária esquerda, e identificaram maior acurácia do último na predição de eventos cardíacos adversos maiores e de trombose dos stents.

\section{O QUE DIZEM AS DIRETRIZES}

O acúmulo de evidências obtidas motivou a comunidade de intervencionistas a propor uma revisão nas diretrizes relativas ao tratamento das lesões de tronco da artéria coronária esquerda, na tentativa de se estabelecer nichos de indicação de intervenção coronária percutânea nesse cenário. ${ }^{20-25,90-92}$ Antes disso, as diretrizes propostas pela Sociedade Brasileira de Cardiologia (SBC), em conjunto com a Sociedade Brasileira de Hemodinâmica e Cardiologia Intervencionista (SBHCl), passaram a classificar a intervenção coronária percutânea em tronco da artéria coronária esquerda não-protegido em indivíduos com elevado risco cirúrgico como classe lla (nível de evidência C), e como classe Ilb nos demais (nível de evidência B). ${ }^{93}$

Recentemente, as diretrizes norte-americanas foram atualizadas e a intervenção coronária percutânea em tronco da artéria coronária esquerda, em indivíduos com anatomia não complexa e de elevado risco cirúrgico, passou a ser considerada opção (classe Ilb, nível de evidência B). Adicionalmente, a indicação prévia de 

no Tronco Não-Protegido da Artéria Coronária Esquerda. Rev Bras Cardiol Invasiva. 2010;18(3):456-67.

reestudo angiográfico protocolar entre 2 e 6 meses após a intervenção coronária percutânea nesses pacientes (classe Ila, nível de evidência C) deixou de vigorar. ${ }^{94}$

À luz das evidências apresentadas, a Sociedade Europeia de Cardiologia também atualizou suas diretrizes (Tabela 5). Pacientes que exibem lesão do tronco da artéria coronária esquerda (óstio ou porção média) acompanhada ou não do acometimento de um outro vaso recebem a classificação lla, nível de evidência B, para a realização da intervenção coronária percutânea. Já aqueles que se encontram em situação semelhante, mas cuja lesão esteja localizada na bifurcação distal do tronco da artéria coronária esquerda, são classificados como Ilb, nível de evidência B. Em indivíduos com escore SYNTAX $\geq 33$ e acometimento de duas ou três artérias além do tronco da artéria coronária esquerda a realização da intervenção coronária percutânea é contraindicada (classe III). ${ }^{95}$

\section{CONCLUSÕES}

A intervenção coronária percutânea em tronco da artéria coronária esquerda é um procedimento factível e seguro, e pode ser oferecida como opção de tratamento para a enfermidade aterosclerótica obstrutiva do tronco da artéria coronária esquerda, desde que consideradas todas as suas peculiaridades. A avaliação do paciente e a discussão com a família acerca das possibilidades de tratamento deverão ser feitas por uma equipe multidisciplinar de cardiologistas clínicos, intervencionistas e cirurgiões cardiovasculares $\mathrm{O}$ uso de stents farmacológicos é mais adequado comparativamente aos não-farmacológicos, considerando-se sobretudo a redução das taxas de novas revascularizações. Não parece haver diferenças substanciais entre as endopróteses eluidoras de sirolimus ou paclitaxel nesse cenário. Embora o uso dos demais stents farmacológicos disponíveis ainda careça de estudos maiores, acreditamos que seus resultados serão semelhantes àqueles obtidos nas demais localizações anatômicas.

A abordagem da bifurcação distal está claramente associada a piores desfechos em comparação ao tratamento do óstio ou do corpo do tronco da artéria coronária esquerda. Quando necessário, o uso de um único stent (T provisional) parece constituir a estratégia mais apropriada. $\mathrm{O}$ uso de dispositivos de imagem intravasculares na orientação do implante, além de poder auxiliar sobremaneira no dimensionamento da endoprótese, parece reduzir desfechos clínicos adversos, sendo recomendado por muitos especialistas. ${ }^{89}$

A seleção dos pacientes pode ser mais bem orientada pela combinação de características clínicas e ana-

TABELA 4

Estudos com utilização de stents farmacológicos na intervenção coronária percutânea em tronco da artéria coronária esquerda (adaptado de Teirstein ${ }^{83}$ )

\begin{tabular}{|c|c|c|c|c|c|c|}
\hline & $\begin{array}{c}\text { Chieffo } \\
\text { et al. }\end{array}$ & $\begin{array}{l}\text { Park } \\
\text { et al. }{ }^{36}\end{array}$ & $\begin{array}{l}\text { Valgimigli } \\
\text { et al.*44 }\end{array}$ & $\begin{array}{c}\text { Price } \\
\text { et al. }{ }^{47}\end{array}$ & $\begin{array}{c}\text { Lee } \\
\text { et al. }{ }^{84}\end{array}$ & $\begin{array}{l}\text { Migliorini } \\
\text { et al. }{ }^{* 85}\end{array}$ \\
\hline Pacientes, $\mathrm{n}$ & 85 & 102 & 95 & 50 & 50 & 101 \\
\hline Bifurcação, \% & 81 & 71 & 65 & 94 & 60 & 87 \\
\hline Mortalidade cardíaca aos 6-12meses, \% & 3,5 & 0 & 11 & 2 & 4 & 11 \\
\hline Seguimento angiográfico, \% & - & 84,3 & - & 98 & 42 & 96 \\
\hline Reestenose angiográfica, \% & 19 & 7 & - & 44 & - & 16 \\
\hline RVA ou RLA, \% & $18,8 \S$ & $2 \rrbracket$ & $6,3 \S$ & $38 \pi$ & $13 \S$ & $14 \S$ \\
\hline
\end{tabular}

TABELA 5

Diretrizes Europeias de intervenção coronária percutânea em tronco da artéria coronária esquerda

\begin{tabular}{lcc}
\hline & RM & ICP \\
\hline Lesão de TCE (isolada ou acompanhada de lesão de 1 vaso, óstio ou corpo) & IA & Ila B \\
Lesão de TCE (isolada ou acompanhada de lesão de 1 vaso, bifurcação) & IA & Ilb B \\
Lesão de TCE + 2 ou 3 vasos, escore SYNTAX $\leq 32$ & IA & IA \\
Lesão de TCE + 2 ou 3 vasos, escore SYNTAX $>32$ & IIIB \\
\hline
\end{tabular}

ICP = intervenção coronária percutânea; RM = cirurgia de revascularização do miocárdio; TCE = tronco da artéria coronária esquerda. 
Attizzani GF, et al. Aspectos Contemporâneos da Intervenção Coronária Percutânea no Tratamento Eletivo de Estenoses Localizadas no Tronco Não-Protegido da Artéria Coronária Esquerda. Rev Bras Cardiol Invasiva. 2010;18(3):456-67.

tômicas, ressaltando-se que à medida que a complexidade avança mais elevadas serão as taxas de desfechos adversos. ${ }^{96}$ Adicionalmente, devemos avaliar criteriosamente a possibilidade de aderência à terapia antiplaquetária dupla, que, embora não tenha sido motivo de discussão neste artigo, desempenha papel fundamental nesses indivíduos.

Finalmente, acreditamos que novos ensaios clínicos prospectivos, randomizados e com poder estatístico adequado para determinar diferenças em desfechos clínicos adversos, comparando a cirurgia de revascularização do miocárdio à intervenção coronária percutânea com o uso de stents farmacológicos de nova geração, devam ser conduzidos (o estudo EXCELL utilizará stents eluidores de everolimus), para que possamos juntar todas as peças desse complexo quebra-cabeças.

\section{AGRADECIMENTO}

Os autores agradecem a Luiz Alberto Mattos pelas valiosas sugestões na confecção do manuscrito.

\section{CONFLITO DE INTERESSES}

Os autores declararam inexistência de conflito de interesses relacionado a este manuscrito.

\section{REFERÊNCIAS}

1. Reig J, Petit M. Main trunk of the left coronary artery: anatomic study of the parameters of clinical interest. Clin Anat. 2004;17(1):6-13.

2. Chaitman BR, Fisher LD, Bourassa MG, Davis K, Rogers WJ, Maynard C, et al. Effect of coronary bypass surgery on survival patterns in subsets of patients with left main coronary artery disease. Report of the Collaborative Study in Coronary Artery Surgery (CASS). Am J Cardiol. 1981;48(4):765-77.

3. Takaro T, Peduzzi P, Detre KM, Hultgren HN, Murphy ML, van der Bel-Kahn J, et al. Survival in subgroups of patients with left main coronary artery disease. Veterans Administration Cooperative Study of Surgery for Coronary Arterial Occlusive Disease. Circulation. 1982;66(1):14-22.

4. Caracciolo EA, Davis KB, Sopko G, Kaiser GC, Corley SD, Schaf $\mathrm{H}$, et al. Comparison of surgical and medical group survival in patients with left main equivalent coronary artery disease: long term CASS experience. Circulation. 1995;91(9): 2335-44.

5. Coles JC, Goldbach MM, Ahmed SN, Wells GA, Mehta HV. Left main-stem coronary artery disease: surgical versus medical management. Can J Surg. 1984;27(6):571-3.

6. O'Keefe JH Jr, Hartzler GO, Rutherford BD, McConahay DR, Johnson WL, Giorgi LV, et al. Left main coronary stenosis: results from the National Registry of Elective Supported Angioplasty. Cathet Cardiovasc Diagn. 1992;25(1):169-73.

7. Stertzer SH, Myler RK, Insel H, Wallsh E, Rossi P. Percutaneous transluminal coronary angioplasty in left main stem coronary stenosis: a five-year appraisal. Int J Cardiol. 1985;9(2):149-59.

8. Tommaso $\mathrm{CL}$, Vogel JH, Vogel RA. Coronary angioplasty in high-risk patients with left main coronary stenosis: results from the National Registry of Elective Supported Angioplasty. Cathet Cardiovasc Diagn. 1992;25(3):169-73.
9. Morice MC, Serruys PW, Sousa JE, Fajadet J, Ban Hayashi E, Perin $M$, et al.; RAVEL Study Group. Randomized study with the sirolimus-coated Bx velocity balloon-expandable stent in the treatment of patients with de novo native coronary artery lesions: a randomized comparison of a sirolimus-eluting stent with a standard stent for coronary revascularization. N Engl J Med. 2002;346(23):1773-80.

10. Moses JW, Leon MB, Popma JJ, Fitzgerald PJ, Holmes DR, O'Shaughnessy C, et al. Sirolimus-eluting stents versus standard stents in patients with stenosis in a native coronary artery. N Engl J Med. 2003;349(14):1315-23.

11. Stone GW, Ellis SG, Cox DA, Hermiller J, O'Shaughnessy C, Mann JT, et al. A polymer-based, paclitaxel-eluting stent in patients with coronary artery disease. N Engl J Med. 2004; 350(3):221-31.

12. Lopez JJ, Ho KK, Stoler RC, Caputo RP, Carrozza JP, Kuntz $\mathrm{RE}$, et al. Percutaneous treatment of protected and unprotected left main coronary stenoses with new devices: immediate angiographic results and intermediate-term follow-up. J Am Coll Cardiol. 1997;29(2):345-52.

13. Silvestri M, Barragan P, Sainsous J, Bayet G, Simeoni JB, Roquebert PO, et al. Unprotected left main coronary artery stenting: immediate and medium-term outcomes of 140 elective procedures. J Am Coll Cardiol. 2000;35(6):1543-50.

14. Black Jr A, Cortina R, Bossi I, Choussat R, Fajadet J, Marco J. Unprotected left main coronary artery stenting: correlates of midterm survival and impact of patient selection. J Am Coll Cardiol. 2001;37(3):832-8.

15. Wong $\mathrm{P}$, Wong V, Tse KK, Chan W, Ko P, Wong CM, et al. A prospective study of elective stenting in unprotected left main coronary disease. Catheter Cardiovasc Interv. 1999;46(2): 153-9.

16. Park SJ, Hong MK, Lee CW, Kim JJ, Song JK, Kang DH, et al. Elective stenting of unprotected left main coronary artery stenosis: effect of debulking before stenting and intravascular ultrasound guidance. J Am Coll Cardiol. 2001;38(3):1054-60.

17. Tan WA, Tamai H, Park SJ, Plokker T, Nobuyoshi M, Suzuki $\mathrm{T}$, et al.; ULTIMA Investigators. Long-term clinical outcomes after unprotected left main trunk percutaneous revascularization in 279 patients. Circulation. 2001;104(14):1609-14.

18. Takagi T, Stankovic G, Finci L, Toutouzas K, Chieffo A, Spanos $\mathrm{V}$, et al. Results and long-term predictors of adverse clinical events after elective percutaneous interventions on unprotected left main coronary artery. Circulation. 2002; 106(6):698-702.

19. Ellis SG, Hill CM, Lytle BW. Spectrum of surgical risk for left main coronary stenosis: benchmark for potentially competing percutaneous therapies. Am Heart J. 1998;135(2 Pt 1):335-8.

20. Taggart DP, Kaul S, Boden W, Ferguson Jr TB, Guyton RA, Mack MJ, et al. Revascularization for unprotected left main stem coronary artery stenosis stenting or surgery. J Am Coll Cardiol. 2008;51(9):885-92.

21. Jonsson A, Hammar N, Nordquist T, Ivert T. Left main coronary artery stenosis no longer a risk factor for early and late death after coronary artery bypass surgery: an experience covering three decades. Eur J Cardiothorac Surg. 2006; 30(2):311-07.

22. Lu JC, Grayson AD, Pullan DM. On-pump versus off-pump surgical revascularization for left main stem stenosis: risk adjusted outcomes. Ann Thorac Surg. 2005;80(1):136-42.

23. Keogh BE, Kinsman R. Fifth National Adult Cardiac Surgical Database: report 2003. Henley on Thames: Dendrite Clinical Systems; 2004.

24. Dewey TM, Magee MJ, Edgerton JR, Mathison M, Tennison D, Mack MJ. Off-pump bypass grafting is safe in patients 
Attizzani GF, et al. Aspectos Contemporâneos da Intervenção Coronária Percutânea no Tratamento Eletivo de Estenoses Localizadas no Tronco Não-Protegido da Artéria Coronária Esquerda. Rev Bras Cardiol Invasiva. 2010;18(3):456-67.

with left main coronary disease. Ann Thorac Surg. 2001; 72(3):788-91

25. Yeatman M, Caputo M, Ascione R, Ciulli F, Angelini GD. Off-pump coronary artery bypass surgery for critical left main stem disease: safety, efficacy and outcome. Eur J Cardiothorac Surg. 2001;19(3):239-44.

26. Smith Jr SC, Dove JT, Jacobs AK, Kennedy JW, Kereiakes D, Kern MJ. ACC/AHA Guidelines for percutaneous coronary intervention (revision of the 1993 PTCA guidelines) - executive summary: a report of the American College of Cardiology/ American Heart Association Task Force on Practice Guidelines (committee to revise the 1993 guidelines for percutaneous transluminal coronary angioplasty) endorsed by the Society for Cardiac Angiography and Intervention. J Am Coll Cardiol. 2001;37(8):2215-38.

27. Sousa JE, Costa MA, Abizaid A, Abizaid AS, Feres F, Pinto $I M$, et al. Lack of neointimal proliferation after implantation of sirolimus-coated stents in human coronary arteries: a quantitative coronary angiography and three-dimensional intravascular ultrasound study. Circulation. 2001;103(2):192-5.

28. Stone GW, Moses JW, Ellis SG, Schofer J, Dawkins K, Morice MC, et al. Safety and efficacy of sirolimus- and paclitaxel-eluting coronary stents. N Engl J Med. 2007;356(10): 998-1008.

29. Tu JV, Bowen J, Chiu M, Ko D, Austin PC, He Y, et al. Effectiveness and safety of drug-eluting stents in Ontario. N Engl J Med. 2007;357(14):1393-402.

30. James SK, Stenestrand U, Lindback J, Carlsson J, Scherstén $\mathrm{F}$, Nilsson $\mathrm{T}$, et al. Long-term safety and efficacy of drugeluting versus bare-metal stents in Sweden. $N$ Engl J Med. 2009;360(19):1933-45.

31. Costa Jr JR, Sousa A, Moreira AC, Costa RA, Cano M, Maldonado $\mathrm{G}$, et al. Incidence and predictors of very late (> 4 years) major cardiac adverse events in the DESIRE-late registry. JACC Cardiovasc Interv. 2010;3(1):12-8.

32. Omerovic E, Ramunddal T, Grip L, Boren J, Matejka G, Albertsson P. In-stent restenosis - benign or dangerous? Clinical presentation of coronary restenosis in Sweden. Circulation. 2008;118 Suppl:973-4.

33. Bainey K, Norris C, Graham M, Ghali W, Knudtson M, Welsh $\mathrm{R}$, et al. Clinical in-stent restenosis with bare-metal stents: is it truly a benign phenomenon? Int J Cardiol. 2008; 128(3):378-82.

34. de Lezo JS, Medina A, Pan M, Delgado A, Segura J, Pavlovic $D$, et al. Rapamycin-Eluting stents for the treatment of unprotected left main coronary artery disease. Am Heart J. 2004;148(3): 481-5.

35. Chieffo A, Stankovic G, Bonizzoni E, Tsagalou E, lakovou I, Montorfano M, et al. Early and mid-term results of drugeluting stent implantation in unprotected left main. Circulation. 2005;111(6):791-5.

36. Park SJ, Kim YH, Lee BK, Lee SW, Lee CW, Hong MK, et al. Sirolimus-eluting stent implantation for unprotected left main coronary artery stenosis: comparison with bare metal stent implantation. J Am Coll Cardiol. 2005;45(3):351-6.

37. Tamburino C, Di Salvo ME, Capodanno D, Marzocchi A, Sheiban I, Margheri M, et al. Are drug-eluting stents superior to bare-metal stents in patients with unprotected non-bifurcational left main disease? Insights from a multicenter registry. Eur Heart J. 2009;30(30):1171-9.

38. Garot $P$, Lefèvre $T$, Savage $M$, Louvard $Y$, Bamlet WR, Willerson J, et al. Nine-month outcome of patients treated by percutaneous coronary interventions for bifurcation lesions in the recent era: a report from the Prevention of Restenosis with Tranilast and its Outcomes (PRESTO) trial. J Am Coll Cardiol. 2005; 46(4):606-12.
39. Costa RA, Mintz GS, Carlier SG, Lansky AJ, Moussa I, Fujii $\mathrm{K}$, et al. Bifurcation coronary lesions treated with the "crush" technique: an intravascular ultrasound analysis. J Am Coll Cardiol. 2005;46(4):599-605.

40. Steingen TK, Maeng M, Wiseth R, Erglis A, Kumsars I, Narbut I, et al. Randomized study on simple versus complex stenting of coronary artery bifurcation lesions. The Nordic Bifurcation Study. Circulation. 2006;114(18):1955-61.

41. Latib A, Colombo A. Bifurcation disease: what do we know, what should we do? JACC Cardiovasc Interv. 2008;1(3):218-26.

42. Colombo A, Bramucci E, Saccà S, Violini R, Lettieri C, Zanini $R$, et al. Randomized study of the crush technique versus provisional side-branch stenting in true coronary bifurcations: the CACTUS (Coronary Bifurcations: Application of the Crushing Technique Using Sirolimus-Eluting Stents) Study. Circulation. 2009;119(1):71-8.

43. Biondi-Zoccai GG, Lotrionte M, Moretti C, Meliga E, Agostoni $\mathrm{P}$, Valgimigli $\mathrm{M}$, et al. A collaborative systematic review and meta-analysis on 1278 patients undergoing percutaneous drug-eluting stenting for unprotected left main coronary artery disease. Am Heart J. 2008;155(2):274-83.

44. Valgimigli $M$, van Mieghem CA, Ong AT, Aoki J, Granillo GA, McFadden EP, et al. Short and long-term clinical outcome after drug-eluting stent implantation for the percutaneous treatment of left main coronary artery disease: insights from the Rapamycin-Eluting and Taxus Stent Evaluated At Rotterdam Cardiology Hospital registries (RESEARCH and T-SEARCH). Circulation. 2005;111(11):1383-9.

45. Godino C, Parodi G, Furuichi S, Latib A, Barbagallo R, Goktekin $\mathrm{O}$, et al. Long-term follow-up (four years) of unprotected left main coronary artery disease treated with paclitaxeleluting stents (from the TRUE registry). Eurolntervention. 2010;5(8):906-16.

46. Toyofuku M, Kimura T, Morimoto T, Hayashi W, Ueda H, Kawai $\mathrm{K}$, et al. Three years outcome after sirolimus-eluting stent implantation for unprotected left main coronary artery disease: insights from the J-CYPHER registry. Circulation. 2009;120(19):1866-74.

47. Price MJ, Cristea E, Sawhney N, Kao JA, Moses JW, Leon MB, et al. Serial angiographic follow-up of sirolimus-eluting stents for unprotected left main coronary artery revascularization. J Am Coll Cardiol. 2006;47(4):871-7.

48. Palmerini T, Marzocchi A, Tamburino C, Sheiban I, Margheri $M$, Vecchi $G$, et al. Impact of bifurcation technique on 2-year clinical outcomes in 773 patients with distal unprotected left main coronary artery stenosis treated with drug-eluting stents. Circ Cardiovasc Interv. 2008;1(3):185-92.

49. Kim YH, Park SW, Hong MK, Park DW, Park KM, Lee BK, et al. Comparison of simple and complex stenting techniques in the treatment of unprotected left main coronary artery bifurcation stenosis. Am J Cardiol. 2006;97(11):1597-601.

50. Vaquerizo $B$, Lefèvre $T$, Darremont $O$, Silvestri $M$, Louvard $\mathrm{Y}$, Leymarie $\mathrm{L}$, et al. Unprotected left main stenting in the real world: two-year outcomes of the French left main taxus registry. Circulation. 2009;119(17):2349-56.

51. Seung KB, Park DW, Kim YH, Lee SW, Lee CW, Hong MK, et al. Stents versus coronary-artery bypass grafting for left main coronary artery disease. N Engl J Med. 2008;358(18):1781-92.

52. Kim YH, Park DW, Lee SW, Yun SC, Lee CW, Hong MK, et al. Long-term safety and effectiveness of unprotected left main coronary stenting with drug-eluting stents compared with bare-metal stents. Circulation. 2009;120(5):400-7.

53. Kim WJ, Park DW, Yun SC, Lee JY, Lee SW, Kim YH, et al. Impact of diabetes mellitus on the treatment effect of percutaneous or surgical revascularization for patients with unprotected left main coronary artery disease: a subgroup 
Attizzani GF, et al. Aspectos Contemporâneos da Intervenção Coronária Percutânea no Tratamento Eletivo de Estenoses Localizadas no Tronco Não-Protegido da Artéria Coronária Esquerda. Rev Bras Cardiol Invasiva. 2010;18(3):456-67.

analysis of the MAIN-COMPARE study. JACC Cardiovasc Interv. 2009;2(10):956-63.

54. Park DW, Seung KB, Kim YH, Lee JY, Kim WJ, Kang SJ, et al. Long-term safety and efficacy of stenting versus coronary bypass grafting for unprotected left main coronary artery disease: 5-year results from the MAIN-COMPARE registry. J Am Coll Cardiol. 2010;56(2):117-24.

55. Han Y, Wang S, Jing Q, Li Y, Liu H, Ma Y, et al. Comparison of long-term efficacy of the paclitaxel-eluting stent versus the bare-metal stent for treatment of unprotected left main coronary artery disease. Am J Cardiol. 2009;103(2):194-8.

56. Buszman PE, Buszman PP, Kiesz R, Bochenek A, Trela B, Konkolewska $M$, et al. Early and long-term results of unprotected left main coronary artery stenting: the LE MANS registry. J Am Coll Cardiol. 2009;54(16):1500-11.

57. Sanmartín M, Baz JA, Claro R, Asorey V, Durán D, Pradas $\mathrm{G}$, et al. Comparison of drug-eluting stents versus surgery for unprotected left main coronary artery disease. Am J Cardiol. 2007;100(6):970-3.

58. Naik H, White AJ, Chakravarty T, Forrester J, Fontana G, Kar S, et al. A meta-analysis of 3773 patients treated with percutaneous coronary intervention or surgery for unprotected left main coronary artery stenosis. JACC Cardiovasc Interv. 2009;2(8):739-47.

59. Pandya S, Young-Hak K, Meyers S, Davidson C, Flaherty J, Park D-W, et al. Drug-eluting versus bare-metal stents in unprotected left main coronary artery stenosis: a meta-analysis. JACC Cardiovasc Interv. 2010;3(6):602-11.

60. Erglis A, Narbute I, Kumsars I, Jagere S, Mintale I, Zakke I, et al. A randomized comparison of paclitaxel-eluting stents versus bare-metal stents for treatment of unprotected left main coronary artery stenosis. J Am Coll Cardiol. 2007;50(6):491-7.

61. Buszman P, Kiesz S, Bochenek A, Peszek-Przybyla E, Szkrobka I, Debinski M, et al. Acute and late outcomes of unprotected left main stenting in comparison with surgical revascularization. J Am Coll Cardiol. 2008;51(5):538-45.

62. Morice MC, Serruys P, Kappetein A, Feldman T, Stahle E, Colombo A, et al. Outcomes in patients with de novo left main disease treated with either percutaneous coronary intervention using paclitaxel-eluting stents or coronary artery bypass graft treatment in the SYNTAX trial. Circulation. 2010; 121(24):2645-53.

63. Kappetein A. SYNTAX: Optimal revascularization strategy in patients with three-vessel disease and/or left main disease: the 2 year outcomes of the SYNTAX trial. [Presented at ESC Congress 2009 September 2; Barcelona, Spain].

64. Valgimigli M, Chieffo A, Lefèvre T, Colombo A, Morice MC, Serruys PW. Revisiting the incidence and temporal distribution of cardiac and sudden death in patients undergoing elective intervention for unprotected left main coronary artery stenosis in the drug-eluting stent era. Eurolntervention. 2007;2(4): 435-43.

65. Chieffo A, Park SJ, Meliga E, Sheiban I, Lee M, Latib A, et al. Late and very late stent thrombosis following drug-eluting stent implantation in unprotected left main coronary artery: a multicenter registry. Eur Heart J. 2008;29(17):2108-15.

66. Mehilli J, Kastrati A, Byrne R, Bruskina O, lijima R, Schulz S, et al.; ISAR-LEFT-MAIN Study Investigators. Paclitaxel versus sirolimus-eluting stents for unprotected left main coronary artery disease. J Am Coll Cardiol. 2009;53(10):1769-72.

67. Sheiban I, Sillano D, Biondi-Zoccai G, Chieffo A, Colombo A, Vecchio $S$, et al. Incidence and management of restenosis after treatment of unprotected left main disease with drugeluting stents: 70 restenotics from a cohort of 718 patients: FAILS (Failure in Left Main Study). J Am Coll Cardiol. 2009; 54(13):1131-6.
68. Meliga E, Garcia-Garcia H, Valgimigli M, Chieffo A, BiondiZoccai G, Maree A, et al. Impact of drug-eluting stent selection on long-term clinical outcomes in patients treated for unprotected left main coronary artery disease: the sirolimus vs paclitaxel drug-eluting stent for left main registry (SP-DELFT). Int J Cardiol. 2009;137(1):16-21.

69. Nakamura S, Nakamura S, Ogawa H, Bae JH, Cahyadi YH, Udayachalerm $W$, et al. Drug-eluting stents for the treatment of left main coronary artery disease with sirolimus, paclitaxel, zotarolimus, biolimus A9, EPC capture and everolimus-eluting stent: multicenter registry in Asia. J Am Coll Cardiol. 2010;55: A181.E1690.

70. Windecker S, Serruys PW, Wandel S, Buszman P, Trznadel $S$, Linke $A$, et al. Biolimus-eluting stent with biodegradable polymer versus sirolimus-eluting stent with durable polymer for coronary revascularization (LEADERS): a randomized noninferiority trial. Lancet. 2008;372(9644):1163-73.

71. Kedhi E, Joesoef KS, McFadden E, Wassing J, Mieghem C, Goedhart D, et al. Second-generation everolimus-eluting and paclitaxel-eluting stents in real-life practice (COMPARE): a randomized trial. Lancet. 2010;375(9710):201-9.

72. Fitzgerald P, Oshima A, Hayase M, Metz J, Bailey S, Baim D, et al. Final results of the Can Routine Ultrasound Influence Stent Expansion (CRUISE) study. Circulation. 2000;102(5): 523-30.

73. Schiele F, Meneveau N, Gilard M, Boschat J, Commeau P, Ming LP, et al. Intravascular ultrasound balloon angioplasty compared with stent: immediate and 6-month results of the multicenter, randomized, Balloon Equivalent to Stent Study (BEST). Circulation. 2003;107(4):545-51.

74. Mudra H, di Mario C, de Jaegere P, Figulla HR, Macaya C, Zahn $\mathrm{R}$, et al. Randomized comparison of coronary stent implantation under ultrasound or angiographic guidance to reduce stent restenosis (OPTICUS Study). Circulation. 2001; 104(12):1343-9.

75. Roy P, Steinberg DH, Sushinsky SJ, Okabe T, Slottow TL, Kaneshige $\mathrm{K}$, et al. The potential clinical utility of intravascular ultrasound guidance in patients undergoing percutaneous coronary interventions with drug-eluting stents. Eur Heart J. 2008;29(15):1851-7.

76. Gerber RT, Latib A, lelasi A, Cosgrave J, Qasim A, Airoldi $F$, et al. Defining a new standard for IVUS optimized drug eluting stent implantation: the PRAVIO study. Catheter Cardiovasc Interv. 2009;74(2):348-56.

77. Costa MA, Angiolillo DJ, Tannenbaum M, Driesman M, Chu A, Patterson J, et al. Impact of stent deployment procedural factors on long-term effectiveness and safety of sirolimuseluting stents (final results of the multicenter prospective STLLR trial). Am J Cardiol. 2008;101(12):1704-11.

78. Park SJ, Kim YH, Park DW, Lee SW, Kim WJ, Suh J, et al.; MAIN COMPARE Investigators. Impact of intravascular ultrasound guidance on long-term mortality in stenting for unprotected left main coronary artery stenosis. Circ Cardiovasc Intervent. 2009;2(3):167-77.

79. Bezerra H, Costa MA, Guagliumi G, Rollins AM, Simon DI. Intracoronary optical coherence tomography: a comprehensive review clinical and research applications. JACC Cardiovasc Interv. 2009;2(11):1035-46.

80. Parodi G, Maehara A, Giuliani G, Kubo T, Mintz GS, Migliorini A. Optical coherence tomography in unprotected left main coronary artery stenting. Eurolntervention. 2010;6(1):94-9.

81. Onuma Y, Girasis C, Piazza N, Garcia-Garcia HM, Kukreja $\mathrm{N}$, Garg S, et al. Long term clinical results following stenting of the left main stem. Insights from RESEARCH and T-SEARCH registries. JACC Cardiovasc Interv. 2010;3(6): 584-94. 
82. Chieffo A, Magni V, Latib A, Maisano F, lelasi A, Montorfano $M$, et al. 5-Year outcomes following percutaneous coronary intervention with drug-eluting stent implantation versus coronary artery bypass graft for the unprotected left main coronary artery lesions: the Milan experience. JACC Cardiovasc Interv. 2010;3(6):595-601.

83. Teirstein PS. Percutaneous revascularization is the preferred strategy for patients with significant left main coronary stenosis. Circulation. 2009;119(7):1021-33.

84. Lee MS, Kapoor N, Jamal F, Czer L, Aragon J, Forrester J, et al. Comparison of coronary artery bypass surgery with percutaneous coronary intervention with drug-eluting stents for unprotected left main coronary artery disease. J Am Coll Cardiol. 2006;46(4):864-70.

85. Migliorini A, Moschi G, Giurlani L, Valenti R, Vergara R, Parodi G, et al. Drug-eluting stent supported percutaneous coronary intervention for unprotected left main disease. Catheter Cardiovasc Interv. 2006;68(2)225-30.

86. Capodanno D, Di Salvo ME, Cincotta G, Miano M, Tamburino C, Tamburino C. Usefulness of the SYNTAX score for predicting clinical outcome after percutaneous coronary intervention of unprotected left main coronary artery disease. Circ Cardiovasc Interv. 2009;2(4):302-8.

87. Capodanno D, Capranzano P, Di Salvo ME, Caggegi A, Tomasello D, Cincotta G, et al. Usefulness of SYNTAX score to select patients with left main coronary artery disease to be treated with coronary artery bypass graft. JACC CardiovasC Interv. 2009;2(8):731-8.

88. Capodanno D, Miano M, Cincotta G, Caggegi A, Ruperto C, Bucato R, et al. EuroSCORE refines the predictive ability of SYNTAX score in patients undergoing percutaneous coronary intervention. Am Heart J. 2010;159(1):103-9.

89. Chen SL, Chen JP, Mintz G, Xu B, Kan J, Ye F, et al. Comparison between the NERS (New Risk Stratification) score and the SYNTAX (Synergy between Percutaneous Coronary Intervention with Taxus and Cardiac Surgery) score in outcome prediction for unprotected left main stenting. JACC CardiovasC Interv. 2010;3(6):632-41.

90. Park SJ, Park DW. Percutaneous coronary intervention with stent implantation versus coronary artery bypass surgery for treatment of left main coronary artery disease. Is it time to change guidelines? Circ Cardiovasc Interv. 2009;2(1):59-68.

91. Stone GW, Mintz GS. Unprotected left main intervention: the light at the end of the tunel? Circ Cardiovasc Intervent. 2009;2(3):156-8.

92. Kandzari DE, Colombo A, Park SJ, Tommaso CT, Ellis SG Guzman LA, et al. Revascularization for unprotected left main disease: Evolution of evidence basis to redefine treatment standards. J Am Coll Cardiol. 2009;54(17):1576-88.

93. Mattos LA, Lemos PA, Rassi A Jr, Marin-Neto JA, Sousa AG, Devito FS, et al. Diretrizes da Sociedade Brasileira de Cardiologia - Intervenção Coronária Percutânea e Métodos Adjuntos Diagnósticos em Cardiologia Intervencionista (II edição 2008). Arq Bras Cardiol. 2008;91(4 Supl 1):1-58.

94. Kushner FG, Hand M, Smith SC Jr, King SB III, Anderson JL, Antman EM, et al. 2009 focused updates: ACC/AHA guidelines for the management of patients with ST elevation myocardial infarction (updating the 2004 guidelines and 2007 focused update) and $\mathrm{ACC} / \mathrm{AHA} / \mathrm{SCAI}$ guidelines on percutaneous coronary intervention (updating the 2005 guideline and 2007 focused update): a report of the American College of Cardiology Foundation/American Heart Association Task Force on Practice Guidelines. J Am Coll Cardiol. 2009;54(23):2205-41.

95. Wijns W, Kohl P, Danchin N, Di Mario C, Falk V, Folliguet $\mathrm{T}$, et al. Guidelines on myocardial revascularization: the Task Force on Myocardial Revascularization of the European Society of Cardiology (ESC) and the European Association for CardioThoracic Surgery (EACTS). Eur Heart J. 2010;31(20):2501-55.

96. Garg S, Stone G, Kappetein AP, Sabik JF $3^{\text {rd }}$, Simonton C, Serruys PW. Clinical and angiographic risk assessment in patients with left main stem lesions. JACC Cardiovas Interv. 2010;3(9):891-901. 